Journal of Applied Pharmaceutical Science Vol. 5 (08), pp. 034-041, August, 2015

Available online at http://www.japsonline.com

DOI: $10.7324 / \mathrm{JAPS} .2015 .50806$

ISSN 2231-3354 (cc)) BY-NC-SA

\title{
Ability of scavenging free radicals and preventing lipid peroxidation of some phenols and ascorbic acid
}

\author{
Jaqueline Badanai, Celi Silva, Denise Martins, Dulce Antunes, Maria Graça Miguel* \\ ${ }^{1}$ Universidade Municipal de São Caetano do Sul, São Paulo, Brasil. ${ }^{2}$ Universidade do Algarve, Faculdade de Ciências e Tecnologia, Departamento de \\ Ciências Biológicas e Bioengenharia, Campus de Gambelas, 8005-139 Faro, Portugal. ${ }^{3}$ Universidade do Algarve, Faculdade de Ciências e Tecnologia, \\ Departamento de Química e Farmácia, Campus de Gambelas, 8005-139 Faro, Portugal.
}

\begin{tabular}{|c|c|}
\hline ARTICLE INFO & ABSTRACT \\
\hline $\begin{array}{l}\text { Article history: } \\
\text { Received on: } 06 / 05 / 2015 \\
\text { Revised on: } 05 / 06 / 2015 \\
\text { Accepted on: } 17 / 06 / 2015 \\
\text { Available online: } 28 / 08 / 2015\end{array}$ & $\begin{array}{l}\text { The capacity for scavenging free radicals and preventing lipid peroxidation of gallic, caffeic, and } p \text {-coumaric } \\
\text { acids, thymol, eugenol and ascorbic acid were evaluated. The capacity for scavenging DPPH }{ }^{\bullet} \text { free radicals were } \\
\text { performed in ethanol } 96 \% \text { and ethanol } 70 \% \text {. In the same assay, the activity estimation was followed at } 10,20 \text { and } \\
30 \text { minutes. In this assay, eugenol presented the best activity (IC } \text { IC }_{50} \text { ranging from } 2.10 \mathrm{mg} / \mathrm{mL} \text { to } 9.74 \mathrm{mg} / \mathrm{mL} \text {. In } \\
\text { the opposite site, } p \text {-coumaric had the lowest activity, in which the } \mathrm{IC}_{50} \text { values were not possible to determine. }\end{array}$ \\
\hline $\begin{array}{l}\text { Key words: } \\
\text { Phenolic acids; eugenol; } \\
\text { thymol; ascorbic acid; } \\
\text { antioxidant. }\end{array}$ & $\begin{array}{l}\text { Generally, } 10 \text { minutes of reaction provided lower scavenging activities than } 30 \text { minutes. The sole exception was } \\
\text { ascorbic acid in which the activities were independent on the time of reaction. Ascorbic acid, eugenol and thymol } \\
\text { possessed higher ability for scavenging DPPH free radicals in ethanol } 70 \% \text { than in ethanol } 96 \% \text {. Gallic and } p \text { - } \\
\text { coumaric acids as well as thymol revealed to be the best scavengers of ABTS }{ }^{\bullet+} \text { free radicals in contrast to } \\
\text { ascorbic acid. The capacity for preventing lipid peroxidation was dependent on the concentration of samples. The } \\
\text { assay showed that higher concentrations of gallic acid, thymol and } p \text {-coumaric acid added to sunflower oil (from } \\
0.3 \text { to } 0.6 \% \text { ) induced higher lipid peroxidation with higher peroxide values. In contrast, increasing the } \\
\text { percentages of caffeic acid and eugenol induced lower peroxidation of the sunflower oil. The percentage of } \\
\text { samples added to this fat did not influence the index of } p \text {-anisidine. In this test, gallic acid had the best capacity } \\
\text { for preventing the formation of } 2,4 \text {-dienals and } 2 \text {-alkenals decadienals able to react with } p \text {-anisidine. }\end{array}$ \\
\hline
\end{tabular}

\section{INTRODUCTION}

Phenols have been reported as being antioxidants and therefore with an important role of protecting organisms against the harmful effects of reactive oxygen species (ROS). These free radicals are very important in the organisms. Oxygen radicals are involved in diverse biochemical activities of great importance in cells such as signal transduction and gene transcription (Uttara $e t$ al., 2009).

In addition, human beings produce oxygen free radicals (superoxide and hydroxyl radicals) and other oxygen reactive species (hydrogen peroxide, nitric oxide, peroxynitrile and hypochlorous acid) due to diverse physiological and biological

\footnotetext{
* Corresponding Author

Maria Graça Miguel, 3Universidade do Algarve, Faculdade de Ciências

e Tecnologia, Departamento de Química e Farmácia, Campus de

Gambelas,8005-139 Faro, Portugal.Email:mgmiguel@ualg.pt
}

processes as a result of aerobic metabolism (Uttara et al., 2009). These free radicals may be responsible for oxidative damage of lipids, proteins and nucleic acids, which can lead to pathologies such as rheumatoid arthritis, diabetics, cancer, atherosclerosis cardiovascular diseases among other chronic and degenerative diseases (Yoshikawa; 1993; Freidovich, 1999; Uttara et al., 2009). However, only an overproduction of free radicals may contribute to the appearance of those diseases. In this way, organisms possess antioxidant defence systems to counteract oxidative damage not only produced by reactive species due normal biochemical and physiological processes but also to radicals of environmental origin (Uttara et al., 2009). When the antioxidant defence systems fail and/or an overproduction of radicals occur such lead to the damage of biomolecules and consequently disease. For this reason, an intake of antioxidants may be necessary which can be supplied through diet. Vitamins $\mathrm{E}$ and $\mathrm{C}, \beta$-carotene and coenzyme $\mathrm{Q}$ are some examples of antioxidants obtained through diet. 
Nevertheless other components also present in foodstuffs of plant origin possess antioxidant activity, as for instance, phenols (Stratil et al., 2006; Pereira et al., 2009; Uttara et al., 2009). The antioxidant ability of phenols is also important in the prevention of food degradation, particularly due to the oxidation of fats. Those components when added to foods may retard rancidity, maintaining their nutritional quality and, consequently increasing their shelf-life (Fukumoto and Mazza, 2000).

Due to the importance of phenols as antioxidants, there is a great interest in these compounds as well as in the methods for the estimation of that property (Dawidowicz and Olszowy, 2012). Such activity may be followed by direct and indirect methods. In the indirect approach, the evaluation of antioxidant activity is based on the capacity for scavenging free radicals. Among the most used indirect methods one can cite the scavenging of DPPH (1,1-diphenyl-2-picrylhydrazyl) and ABTS ${ }^{\bullet+}$ [2,2'-azinobis(3-ethylbenzothiazoline-6-sulfonic acid)] free radicals (Dawidowicz and Olszowy, 2012). Such methods are not associated with the real oxidative degradation (Roginsky and Lissi, 2005).

Direct methods are based on the effect of the sample with potential antioxidant activity on the oxidative degradation of a tested substrate (individual lipids, lipid mixtures, proteins, nucleic acids, lipid containing biologically relevant species such as blood plasma, lipoproteins of low density, biological membranes, among other substrates) (Roginsky and Lissi, 2005). Lipid oxidation is generally evaluated determining the peroxide value thiobarbituric acid reactive substances (TBARS), conjugated dienes, anisidine value or checking volatile compounds (Kristinová et al., 2009).

Generally indirect methods are mostly used, such as DPPH and ABTS methods, although their poor repeatability and the lack of certitude for the capability of samples to inhibit oxidative processes. Reagent concentrations, time of incubation, solvents used, matrix of sample, among other factors may be determinant on the results obtained which may vary strongly, do not permitting compare results (Roginsky and Lissi, 2005; PérezJiménez and Saura-Calixto, 2006).

Although direct methods are more adequate, particularly those based on the model of the chain controlled reaction, they are generally time-consumed methodologies and therefore they are not suitable for routine testing natural products (Roginsky and Lissi, 2005). These authors even consider that direct methods may be recommended to use for calibrating indirect methods.

In the present work the capacity of cinnamic acid derivatives ( $p$-coumaric acid and caffeic acid), benzoic acid derivative (gallic acid), phenylpropene (eugenol), aromatic monoterpene (thymol) and ascorbic acid (vitamin C) for scavenging DPPH and $\mathrm{ABTS}^{\circ+}$ free radicals were evaluated. The type of solvent and course time reaction in DPPH method was also assayed. At the same time, the capacity of the same compounds for preventing lipid peroxidation was also estimated, using sunflower oil as lipidic substrate.

\section{MATERIAL AND METHODS}

\section{Scavenging free radical ability}

1,1-Diphenyl-2-picrylhydrazyl (DPPH) free radical-scavenging activity

A $25 \mu \mathrm{L}$ aliquot of each sample dissolved in $96 \%$ ethanol or ethanol $70 \%$ was mixed with $975 \mu \mathrm{L}$ DPPH $60 \mu \mathrm{M}$ dissolved in $96 \%$ ethanol or ethanol $70 \%$, respectively. After 10, 20 or $30 \mathrm{~min}$ reaction period, the absorbance (Abs) was recorded at $517 \mathrm{~nm}$ (Brand-Williams et al., 1995). Several sample concentrations were prepared for the determination of the percentage of inhibition that was calculated using a graph of the scavenging effect percentage against sample concentrations. The sample concentration providing $50 \%$ inhibition $\left(\mathrm{IC}_{50}\right)$ was calculated from that graph. The scavenging effect percentage was calculated from the formula: Percentage of inhibition $=\left[\left(\mathrm{Abs}_{\text {control }}-\mathrm{Abs}_{\text {sample }}\right) / \mathrm{Abs}_{\text {control }}\right] \times 100$.

In the control, the volume of sample is replaced by the same volume of solvent. The percentage was plotted against the samples, and $\mathrm{IC}_{50}$ values were estimated (therefore, concentration of samples able to scavenger $50 \%$ of the DPPH free radical.

\section{2,2'-Azino-bis(3-ethylbenzothiazoline-6-sulphonic acid (ABTS) free radical-scavenging activity}

The ABTS radical was generated by reaction of a $7 \mathrm{mM}$ ABTS aqueous solution with $\mathrm{K}_{2} \mathrm{~S}_{2} \mathrm{O}_{8}(2.45 \mathrm{mM})$ in the dark for 16 $\mathrm{h}$ and adjusting the absorbance at $734 \mathrm{~nm}$ to 0.7 at room temperature $(\operatorname{Re}$ et al., 1999). Samples $(10 \mu \mathrm{L})$ were added to $990 \mu \mathrm{L}$ of ABTS and the absorbance at $734 \mathrm{~nm}$ was read after 6 min (Antunes et al., 2010). Several sample concentrations were prepared and the percentage inhibition was calculated from the formula:

Percentage of inhibition $=\left[\left(\mathrm{Abs}_{\text {control }}-\mathrm{Abs}_{\text {sample }}\right) / \mathrm{Abs}_{\text {control }}\right] \times 100$.

$\mathrm{IC}_{50}$ values (concentration of sample able to scavenger $50 \%$ of the ABTS free radical) were determined as reported in DPPH method. In the control, the volume of sample is replaced by the same volume of solvent.

\section{Sample preparation for the estimation of lipid oxidation}

Four hundred and fifty or nine hundred milligrams of each sample were added separately to $150 \mathrm{~g}$ of the sunflower oil. Each mixture was stirred for $5 \mathrm{~min}$ at room temperature, placed in closed transparent glass bottles $(250 \mathrm{~mL})$, at $60^{\circ} \mathrm{C}$, and kept in the dark. After 26 days, the peroxide value and $p$-anisidine were evaluated as reported below.

\section{Peroxide value}

A mixture of sunflower oil and chloroform/acetic acid $(3: 2)(\mathrm{v} / \mathrm{v})$ was left to react in darkness with saturated potassium iodine solution. The free iodine was titrated with a sodium thiosulphate solution $(0.01 \mathrm{M})$. Peroxide value was determined in milliequivalents of active oxygen per kilogram of oil $\left(\mathrm{mEq} \mathrm{O}_{2} / \mathrm{kg}\right)$, 
according to the following formula: $\mathrm{Ip}=10 \times\left(\mathrm{n}_{1}-\mathrm{n}_{2}\right) / \mathrm{m}$ where $\mathrm{n}_{1}$ was the $\mathrm{mL}$ of sodium thiosulphate consumed in sample titration, $\mathrm{n}_{2}$ was the $\mathrm{mL}$ of sodium thiosulphate consumed in blank titration and $m$ was the amount of sample in $g$ (Neves et al., 2011).

\section{p-Anisidine value}

$p$-Anisidine value was determined as reported previously (Neves et al., 2011). Briefly, it was carried out by the determination of the absorbance increase, measured at $350 \mathrm{~nm}$, of a sample solution of $0.5 \mathrm{~g}$ of sunflower oil $(\mathrm{m})$ in iso-octane $(25 \mathrm{~mL})$, before $\left(\mathrm{A}_{1}\right)$ and after reaction with $p$-anisidine $\left(\mathrm{A}_{2}\right)$ in glacial acetic acid in the dark. The $p$-anisidine value was determined with the following formula: $25 \times\left(1.2 \mathrm{~A}_{2}-\mathrm{A}_{1}\right) / \mathrm{m}$.

\section{Statistical Analysis}

Each treatment consisted of 3 replications. Statistical analysis was carried out with the SPSS 20.0 software (IBM, Inc.). Two-way ANOVA and Duncan's Multiple-Range Test $(\mathrm{P}<0.05)$ for comparisons among treatments over time was performed.

\section{RESULTS AND DISCUSSION}

\section{Scavenging free radical ability}

TEAC (Trolox Equivalent Antioxidant Capacity) assay is also known as ABTS [2,2'-azinobis-(3-ethylbenzothiazoline-6sulphonate)] radical cation $\left(\mathrm{ABTS}^{\circ+}\right.$ ) method. ABTS is a compound that after oxidation by peroxyl radicals or other oxidants originates the radical cation $\mathrm{ABTS}^{\circ+}$, which is blue and absorbs light at $734 \mathrm{~nm}$. An antioxidant reacts directly with the radical cation $\mathrm{ABTS}^{\bullet+}$, reducing the blue colour (Prior et al., 2005). DPPH $^{\bullet}$ is a very stable organic nitrogen radical of deep purple colour, which absorbs light at $515 \mathrm{~nm}$. There is a loss of colour when this radical reacts directly with an antioxidant (Prior et al., 2005).

Those two methods were used for evaluating the antioxidant ability of $p$-coumaric and caffeic acids (both cinnamic acid derivatives), gallic acid (benzoic acid), eugenol (phenylpropanoid), thymol (phenolic monoterpene) and ascorbic acid (vitamin). These compounds were chosen based on their known antioxidant capacity, albeit their different chemical structures.

$p$-Coumaric acid had the best capacity for scavenging the radical cation $\mathrm{ABTS}^{\bullet+}$, nevertheless the worst one for scavenging $\mathrm{DPPH}^{\bullet}$ radicals (Table 1 ). Such results are in accordance to those already reported by several authors (Nenadis and Tsimidou, 2002; Nenadis et al., 2004; Kristinová et al., 2009; Kiliç and Yeşilouğlou, 2013; Xie and Schaich, 2014).

$p$-Coumaric acid, thymol and gallic acid presented the best capacity for scavenging $\mathrm{ABTS}^{\bullet+}$. $p$-Coumaric acid also presented better capacity for scavenging $\mathrm{ABTS}^{\bullet+}$ than caffeic and ascorbic acids according to the results previously reported by $\operatorname{Re} e t$ al. (1999). The results obtained in our work did not coincide with those found when $\mathrm{DPPH}^{\bullet}$ method was used for evaluating the capacity for scavenging free radicals (Table 1). In DPPH assay, $p$ coumaric acid was the worst compound for scavenging $\mathrm{DPPH}^{\bullet}$, not even being possible to determine $\mathrm{IC}_{50}$ values, whereas in TEAC method, the same phenolic compound was one of the best scavengers of $\mathrm{ABTS}^{\bullet+}$. The $\mathrm{IC}_{50}$ values found for $p$-coumaric acid, thymol and gallic acid were lower when compared to those obtained in DPPH method, such meaning better antioxidant activity when measured through TEAC method. In contrast, the $\mathrm{IC}_{50}$ values found for ascorbic acid were practically similar to those in which the DPPH method used ethanol $70 \%$ as solvent (around $40 \mathrm{mg} / \mathrm{mL}$ ) (Table 1). Eugenol had worse ability for scavenging $\mathrm{ABTS}^{\circ+}$ than $\mathrm{DPPH}^{\bullet}$, according to the $\mathrm{IC}_{50}$ values found for this compound in both methods (Table 1).

The different trend of antioxidant activity observed in the present work was also reported by Khanan et al. (2012), when they evaluated antioxidant abilities of diverse leafy vegetables. Those authors reported that the presence of different phenols in the samples and not identified in their work, could contribute to such results. According to the experiments of Arts et al. (2004) with pure flavonoids, they reported that some products resulting from the oxidation of flavonoids in the presence of $\mathrm{ABTS}^{\bullet+}$ had higher antioxidant capacity, reacting faster with $\mathrm{ABTS}^{\circ+}$ than with the parent compound. Other example given by the same authors was the trolox quinone formed during scavenging $\mathrm{ABTS}^{\bullet+}$ from trolox, which did not react with $\mathrm{ABTS}^{\circ+}$. According to these results, the authors suggested that TEAC assay did not necessarily reflect the antioxidant ability of only one structure, that is, the reaction products may have a considerable contribution to the TEAC method.

However, the same has also been reported in DPPH method: the formation of complex mixtures of dimeric compounds originated by different coupling processes as observed for eugenol, caffeic acid, among other compounds, which also possesses capacity for acting with $\mathrm{DPPH}^{\bullet}$ free radicals (Bortolomeazzi et al., 2010; Gülçin et al., 2012). According to these findings, the ability of the polyphenols to act as antioxidants not only depends on the redox properties of their phenolic hydroxyl groups and the potential for electron delocalization across the chemical structure, as also of the possible reactivity of the reaction products formed during the reaction with DPPH and ABTS. For this reason, some authors consider that this contribution of the reaction products to the radical scavenging activity limits those methods to evaluate structure-activity relationship as well as correlate the results with the antioxidant activity obtained by other methods (Bortolomeazzi et al., 2010). In fact and only considering the ABTS and DPPH methods used in the present work, it is very difficult to explain the results obtained in both methods, since there is no correlation of activities between the two methods.

However, some authors had reported that compounds with lower redox potential possess higher antioxidant activity and therefore they considered that redox potentials could be considered as a good measure of antioxidant activity (Teixeira et al., 2013). In our case, such is not as evident. 
According to Kristinová et al. (2009) the different capacity of phenolic compounds to scavenge free radicals depending on the method used may be due to the specific reactions between the different assay reagents and the antioxidant samples and unrelated reactions of phenols (dimerization, polymerization) that occur during the reactions.

Kiliç and Yeşilouğlou (2013) found that the concentration $30 \mu \mathrm{g} / \mathrm{mL}$ had $55.6 \%$ capacity for scavenging DPPH radicals. In our case, only from $1.84 \mathrm{mg} / \mathrm{mL}$ the capacity for scavenging DPPH radicals was $>50 \%(50.7 \%)$, when the solvent of DPPH radicals was ethanol $70 \%$ (Fig. 1). When the solvent was ethanol $96 \%$, higher concentration of $p$-coumaric was needed to reach $50 \%$ activity $(2.46 \mathrm{mg} / \mathrm{mL})$ (Fig. 2$)$.

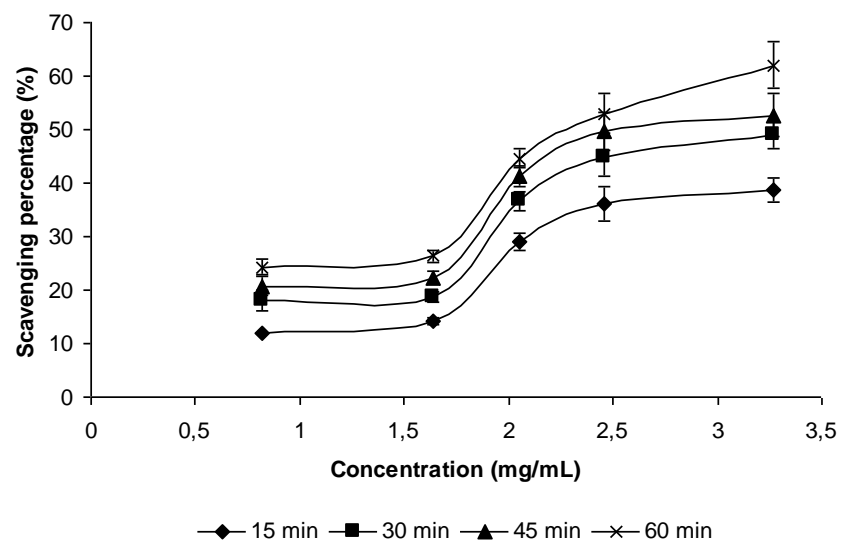

Fig. 1: DPPH scavenging ability of $p$-coumaric acid when the assay was carried out in ethanol $96 \%$.

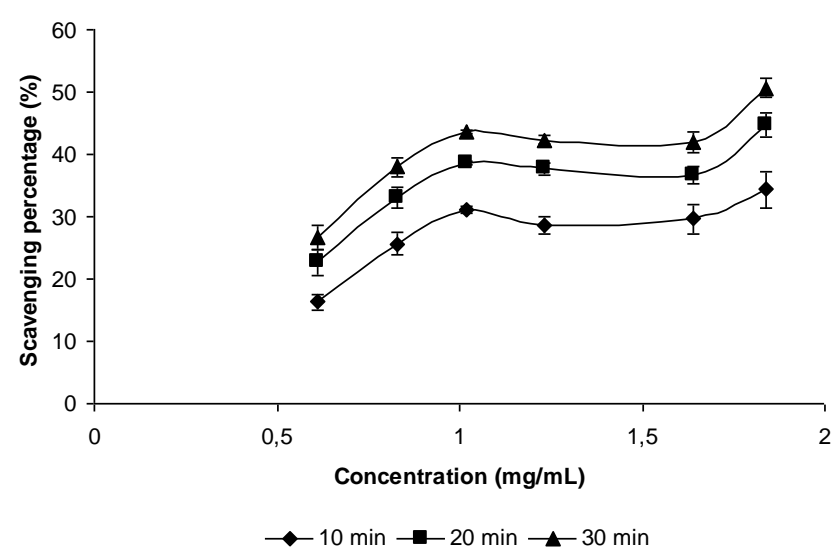

Fig. 2: DPPH scavenging ability of $p$-coumaric acid when the assay was carried out in ethanol $70 \%$.

In addition, these activities were highly dependent on the reaction time (Fig. 1 and 2). $p$-Coumaric acid is a mono-hydroxyl derivative of cinnamic acid and according to some authors, aromatic acids containing only one $\mathrm{OH}$-group does not react with DPPH (Roginsky and Lissi, 2005; von Gadow et al., 1997) or never react with more than $75 \%$ of the initial DPPH radicals, even after several hours of reaction time and at very high concentrations (Brand-Williams et al., 1995). These authors considered, therefore, that this phenolic compound presents a slow kinetic behaviour. Our results demonstrated that the activity of $p$-coumaric for scavenging $\mathrm{DPPH}^{\bullet}$ radicals depend on the concentration, type of solvent used and reaction time (Fig. 1 and 2).

Thymol was the second worst phenolic compound for scavenging $\mathrm{DPPH}^{\bullet}$ radicals (Table 1 ) and, such as $p$-coumaric acid, its activity was dependent on the type of solvent and reaction time (Fig 3 and 4). Antioxidant activity of thymol after 30 minutes of reaction was better than that determined soon after 10 minutes (Table 1). Thymol also had better capacity for scavenging $\mathrm{DPPH}^{\bullet}$ free radicals when the solvent ethanol $70 \%$ for solubilise $\mathrm{DPPH}^{\circ}$ was used than ethanol $96 \%$ (Table 1). Without significant differences, thymol had similar capacity for scavenging radical cation $\mathrm{ABTS}^{\bullet+}$ to that found for $p$-coumaric acid (Table 1).

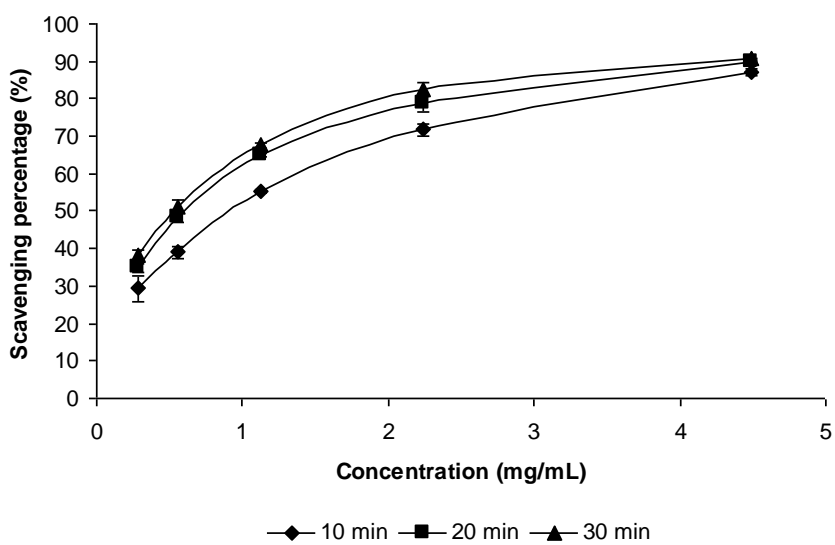

Fig. 3. DPPH scavenging ability of thymol when the assay was carried out in ethanol $96 \%$.

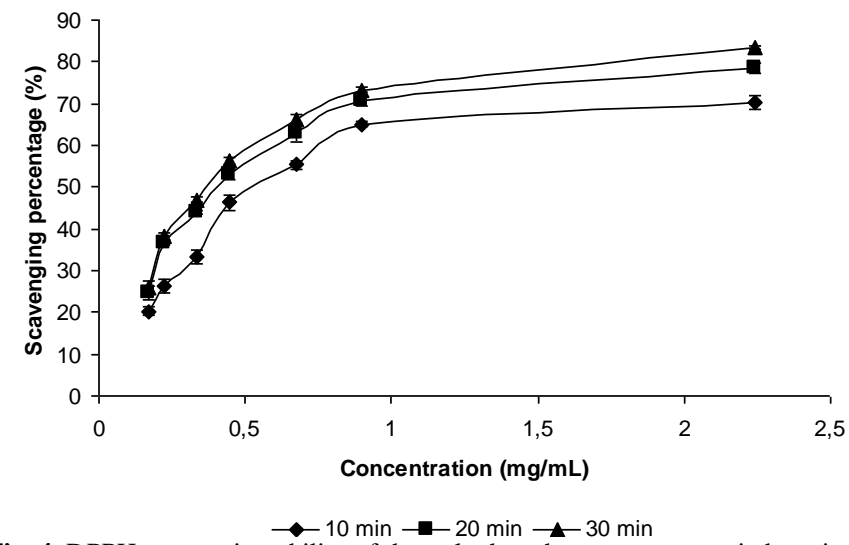

Fig. 4. DPPH scavenging ability of thymol when the assay was carried out in ethanol $70 \%$.

The main methods for evaluating in vitro antioxidant activities are grouped in two categories: hydrogen atom transfer (HAT) reaction and electron transfer (ET) reaction-based methods. For example, ORAC (oxygen radical absorbance capacity) assay measures the capacity of an antioxidant for scavenging peroxyl radicals $\left(\mathrm{ROO}^{\circ}\right)$ by a hydrogen atom transfer reaction. TEAC (Trolox equivalent antioxidant capacity) and 2,2-diphenyl-1picrylhydrazyl (DPPH') methods have been classified as SET reactions (Prior et al., 2005). Nevertheless, these free radicals may react with antioxidants either by direct reduction via electron transfer or by hydrogen atom transfer reaction (Jiménez et al., 
2004; Prior et al., 2005). The contribution of a direct abstraction of phenol H-atom by the free radical (DPPH ${ }^{\circ}$ ) (HAT reaction) or of an electron-transfer process from the phenol $(\mathrm{ArOH})$ or its phenoxide $\left(\mathrm{ArO}^{-}\right)$to the free radical $\left(\mathrm{DPPH}^{*}\right)$ (ET reaction) depends on the nature of the solvent and/or the redox potentials of the species involved (Foti et al., 2004). Generally, HAT mechanisms predominate in apolar solvents, but with very strong oxidizing radicals $\left(\mathrm{Cl}_{3} \mathrm{COO}\right)^{\circ}$, ET may be the preferential mechanism even in apolar solvents (Foti et al., 2004). According to some authors (Foti et al., 2004; Foti et al., 2008), the best capacity of esters of cinnamic acids than cinamic acid derivatives for scavenging $\mathrm{DPPH}^{*}$ radicals is due to the fact that dissociation of the carboxylic group reduces the quantity of phenoxide $\left(\mathrm{ArO}^{-}\right)$ in equilibrium with the phenol ( $\mathrm{ArOH})$. This fact may partly explain the weak capacity of $p$-coumaric acid to react with $\mathrm{DPPH}^{\circ}$ in contrast to the remaining samples assayed (Table 1).

SET and HAT mechanisms may occur together being the balance determined by antioxidant structure and $\mathrm{pH}$ (Prior et al., 2005; Karadag et al., 2009). In DPPH method, several works have demonstrated that the mechanism involving hydrogen atom transfer may occur in at least four different sequences: hydrogenatom transfer (HAT), proton-coupled electron transfer (PCET), sequential proton-loss electron transfer (SPLET), and electrontransfer proton-loss (ET-PT) (Foti et al., 2008). These authors consider that the reaction between esters of cinnamic acid or cinnamic acid derivatives and DPPH ${ }^{\circ}$ occur by SPLET in solvents with high dielectric constant. The reaction between phenols and $\mathrm{DPPH}^{\circ}$ may occur by a combination of PC-ET and SPLET mechanisms. PC-ET dominates in non-polar solvents of low dielectric constant and of low basicity, while SPLET mechanism occurs preferentially in alcohols such as methanol or ethanol, which have higher dielectric constant and high ability to solvate phenolic anions.

PC-ET is a slower mechanism than SPLET one (Dawidowicz and Olszowy, 2012). Higher dielectric constant of water than ethanol may me responsible for the best capacity of $p$ coumaric or thymol for scavenging DPPH ${ }^{*}$ radicals in the solvent ethanol $70 \%$. In addition, this mixture probably has better capacity for solvating phenolic anions. The combination of these two factors may partly explain the best antioxidant activities found when $\mathrm{DPPH}^{\circ}$ radicals were solubilised in the solvent ethanol $70 \%$. Eugenol and gallic acid presented the best capacity for scavenging $\mathrm{DPPH}^{\bullet}$ radicals (Table 1 ). When the solvent assayed was ethanol $96 \%$, the reaction time did not influence the activity of eugenol (Fig. 5).

Nevertheless when ethanol $70 \%$ was used as solvent, activity differences were obtained, after 10 minutes of reaction the activity was worse than 20 and 30 minutes (Fig. 6, Table 1). Such results demonstrated that the behaviour of eugenol in the presence of $\mathrm{DPPH}^{\bullet}$ radicals is dependent on the solvent. Such as reported for $p$-coumaric acid and thymol, in eugenol, the presence of water ameliorated its activity, maybe due to the higher dielectric constant of water. The capacity for scavenging $\mathrm{DPPH}^{*}$ radicals of phenolic compounds increases with increasing degree of hydroxylation (Rice-Evans et al., 1996; Scherer and Godoy, 2009). Such may partly explain the worst activity of thymol and $p$-coumaric acid with only one $-\mathrm{OH}$ attached to the aromatic ring. Nevertheless eugenol with also only one $-\mathrm{OH}$ presented the best activity. Such means that other groups attached to the aromatic ring and their positions have a role in the antioxidant activity. The electron donating groups, such as $-\mathrm{CH}_{2}-\mathrm{CH}=\mathrm{CH}$ and $-\mathrm{OCH}_{3}$ attached to the aromatic ring of eugenol in positions $p$ - and $o$-, respectively seem to contribute to the best activity found for this phenolic compound when measured through the DPPH method (Table 1). Methoxyl groups in an ortho-position to a hydroxyl group resulted in an increase of the antioxidant activity in studies performed by Teixeira et al. (2013).

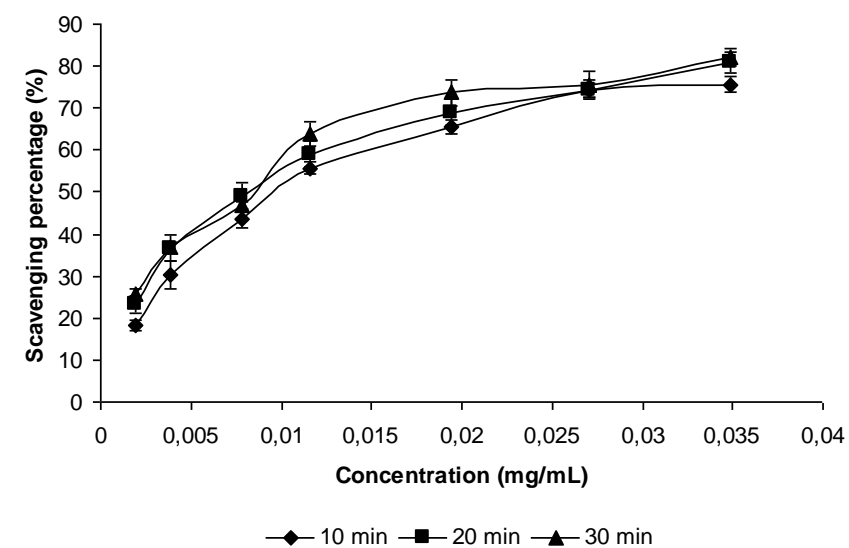

Fig. 5. DPPH scavenging ability of eugenol when the assay was carried out in ethanol $96 \%$.

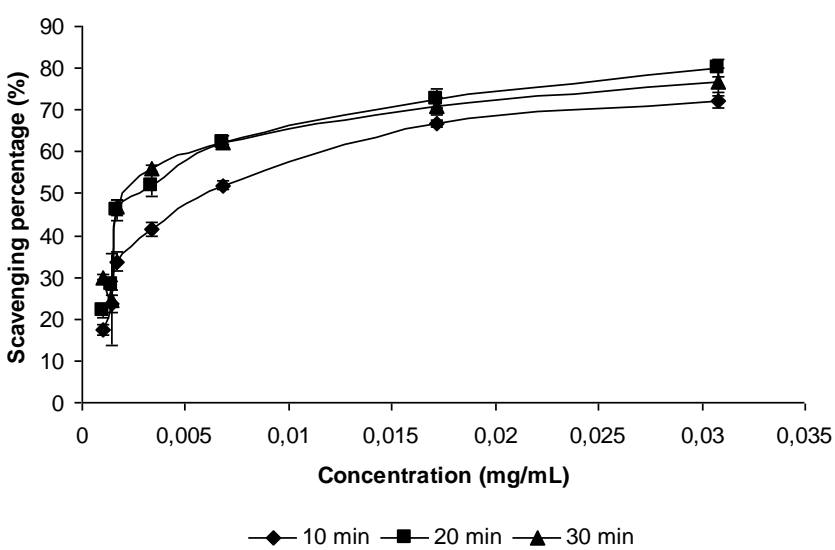

Fig. 6. DPPH scavenging ability of eugenol when the assay was carried out in ethanol $70 \%$

In the case of gallic acid, the effect of 3-OH attached to the aromatic ring superposes to the electron withdrawing of $\mathrm{COOH}$, which makes it stronger as scavenger than $p$-coumaric and caffeic acids.

Ascorbic acid is not an aromatic compound but presents a good capacity for scavenging DPPH ${ }^{\bullet}$ radicals (Table 1). Such activity and according to Nenadis et al. (2007) may be attributed to the possible formation of a quinone from its ene-diol group by donating two hydrogen atoms to the free radical. 
Table 1: Antioxidant activity of some phenols and ascorbic acid expressed as IC50 (mg/mL) for DPPH and ABTS method, and as mEq O2/kg for peroxide value, and index of p-anisidine.

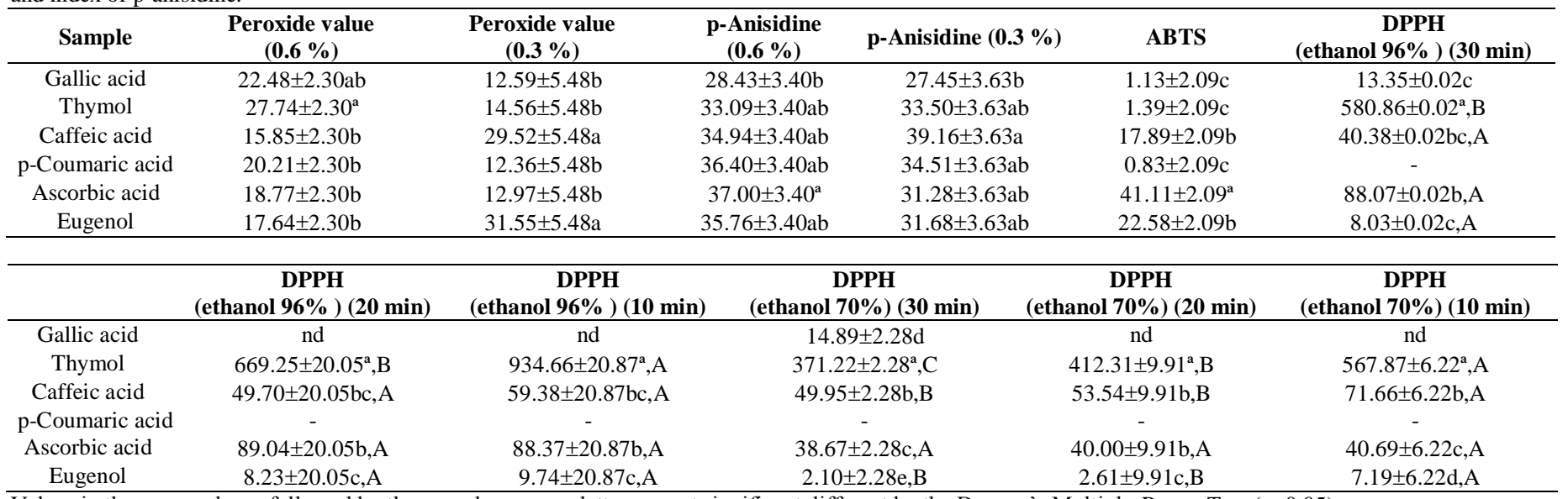

Values in the same column followed by the same lower case letter are not significant different by the Duncan's Multiple-Range Test (p<0.05).

For DPPH analysis, values in the same row followed by the same upper case letter are not significant different by the Duncan's Multiple-Range Test ( $\mathrm{p}<0.05)$.

In contrast to the remaining compounds, the capacity for scavenging $\mathrm{DPPH}^{\bullet}$ radicals was independent on the reaction time (Fig. 7 and 8 ).

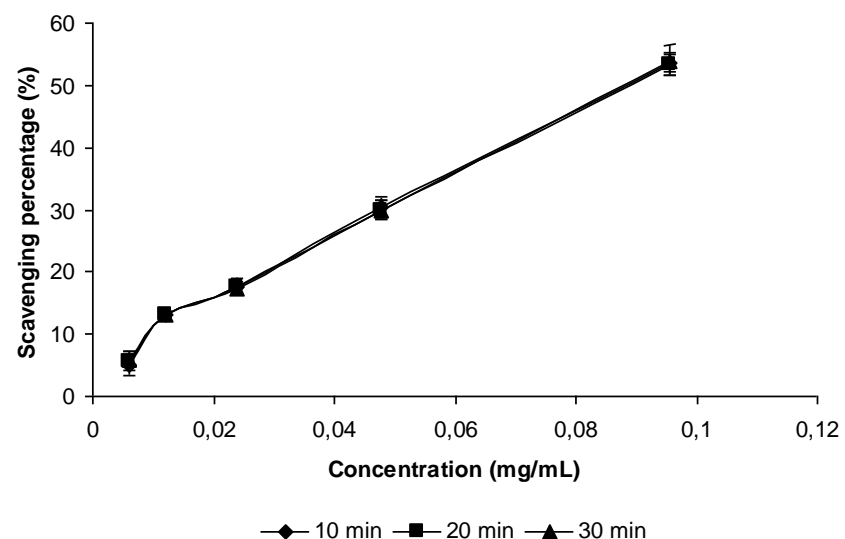

Fig. 7. DPPH scavenging ability of ascorbic acid when the assay was carried out in ethanol $96 \%$.

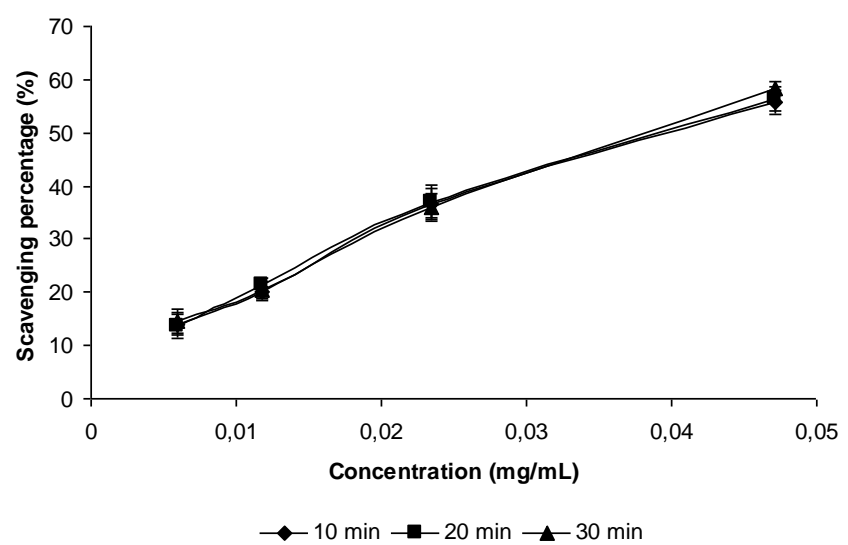

Fig. 8. DPPH scavenging ability of ascorbic acid when the assay was carried out in ethanol $70 \%$.

This behavior was observed in both solvents; nevertheless the activity of vitamin $\mathrm{C}$ was significantly better when the solvent used was ethanol $70 \%$ such as for thymol and eugenol. In ethanol $70 \%$, ascorbic acid was even better antioxidant than caffeic acid (Table 1). The best activity of ascorbic acid in ethanol $70 \%$, such as observed for thymol and $p$-coumaric acid, may also be explained by the SPLET mechanism induced by the proton transfer of the ascorbic acid to the solvent, as reported above for thymol and $p$-coumaric acid.

\section{Lipid oxidation}

The values presented in peroxide and $p$-anisidine values are those obtained after 26 days of fat storage at $60^{\circ} \mathrm{C}$, whereas the remaining results are presented as $\mathrm{IC}_{50}$ values (Table 1).

For a concentration of $0.6 \%$ of samples added to the sunflower oil, caffeic acid, eugenol, ascorbic and $p$-coumaric acids presented the best capacity for preventing the formation of hydroperoxides. Nevertheless, after the formation of these oxidation intermediates, these compounds are not sufficiently strong to prevent the formation of the secondary oxidation products such as 2,4-dienals and 2-alkenals decadienals, measured through the $p$-anisidine method (Table 1). In this case, gallic acid reveals to be the most effective (Table 1). However, some care must be taken with gallic acid, because some authors detected that this phenol compound exhibit significant prooxidant effect on total oxidation in the pH 3.0 emulsions (Osborn and Akoh, 2003). In fact, in our case, when the concentration of this benzoic acid derivative was doubled (from $0.3 \%$ to $0.6 \%$ ), the formation of hydroperoxides also increased (from 12.59 to $22.81 \mathrm{mEq} \mathrm{O}_{2} / \mathrm{kg}$, respectively) (Table 1). Other phenolic compounds also presented this trend: $p$-coumaric acid (from 12.36 to $20.21 \mathrm{mEq} / \mathrm{O}_{2} \mathrm{~kg}$, respectively), and thymol (from 14.56 to $27.84 \mathrm{mEq} / \mathrm{O}_{2} \mathrm{~kg}$, respectively). There are some examples that show that some phenols considered antioxidants may have pro-oxidant activity in high concentrations or in the presence of metal ions (Bouayed and Bohn, 2010; Yordi et al., 2012). On the contrary, increasing the amounts of caffeic acid or eugenol in sunflower oil, the peroxidation of the vegetable oil decreased. The peroxide values 
found were 29.52 and $15.85 \mathrm{mEq} \mathrm{O}_{2} / \mathrm{kg}$, for caffeic acid at 0.3 and $0.6 \%$, respectively; and for eugenol, the values found were 31.55 and $17.64 \mathrm{mEq} \mathrm{O}_{2} / \mathrm{kg}$ (Table 1). These results suggest an antioxidant ability of these two phenols, in contrast to $p$-coumaric and gallic acids, and thymol.

The capacity of $p$-coumaric acid for preventing lipid peroxidation was already reported by Kiliç and Yeşilouğlou (2013), although using a different method. The inhibition of lipid peroxidation was determined according to the thiocyanate method and using linoleic acid emulsion in potassium phosphate buffer as lipidic substrate. They found that $p$-coumaric acid was better than the controls butylated hydroxyanisole (BHA) and butylated hydroxytoluene (BHT).

In bulk methyl linoleate or in fish muscle, Kikuzaki et al. (2002) and Medina et al. (2007), respectively, reported that caffeic acid had the capacity for preventing the formation of hydroperoxides, in contrast to $p$-comaric acid which had a very weak capacity, as observed in the DPPH method or in the ferric reducing/antioxidant power (FRAP). Such results should be expected since both methods utilize the same single-electron transfer mechanism, according to Medina et al. (2007). Using other lipidic substrate such as egg yolk phosphatidylcholine liposomes which hydroperoxides were induced by AAPH [2,2'azobis(2-amidinopropane) dihydrochloride], Kikuzaki et al. (2002) found that gallic acid had the lowest capacity for preventing hydroperoxides immediately followed by caffeic acid and $p$ coumaric acid. In this lipid substrate, ascorbic acid had lower capacity for preventing lipid peroxidation than gallic acid. This is not coincident with our results, in which $p$-coumaric acid was a very weak scavenger of $\mathrm{DPPH}^{\bullet}$ free radicals, but with similar capacity to those of caffeic and ascorbic acids for preventing hydroperoxide formation when their concentration in sunflower oil was $0.6 \%$ (Table 1 ). Such results are somehow different to those found in our experimental work, but such may partly be explained by the type of lipid substrate.

\section{CONCLUSIONS}

Several methods may be assayed for evaluating the antioxidant activity of samples; nevertheless diverse factors may contribute to originate distinct results. In the present work, the same assay (DPPH method) but using different solvents led to different results. Time of reaction also produced diverse results. When comparing two different methods but both based on the capacity for scavenging free radicals $\left(\mathrm{DPPH}^{\bullet}\right.$ or $\mathrm{ABTS}^{\bullet+}$ ) the results were not coincident. Some authors, as reported in the Introduction, suggest that methods based on the lipid oxidation may be recommended to use for calibrating those methods which are based on scavenging free radicals. In the present work such was not as evident because some of the compounds with good capacity for preventing hydroperoxide formation were not the same which possessed the best capacity for scavenging some free radicals. For example, eugenol, was the best antioxidant in terms of $\mathrm{DPPH}^{\bullet}$ scavenging ability, whereas $p$-coumaric acid, thymol and gallic acid were the best compounds for scavenging ABTS free radicals. These compounds were also the best ones for preventing hydroperoxidation of a lipidic substrate but along with caffeic acid.

Higher concentrations of thymol, gallic acid and $p$ coumaric acid induced higher peroxidation of triglycerides of sunflower oil than at lower concentrations. On the contrary, caffeic acid and eugenol presented better capacity for preventing peroxidation at higher concentration than at lower ones. Several factors (methods, concentrations, matrices, among others) determine different results which need be carefully understood and enlightened.

\section{Conflicts of interest}

All authors declare no conflicts of interest.

\section{ACKNOWLEDGEMENTS}

Authors are grateful to FCT-Fundação para a Ciência e Tecnologia (PEst-OE/EQB/LA0023/2013).

\section{REFERENCES}

Antunes M.D.C., Dandlen S., Cavaco A.M., Miguel G., Effects of postharvest application of 1-MCP and post-cutting dip treatment on the quality and nutritional properties of fresh-cut kiwifruit. J. Agric. Food Chem. 2010; 58:6173-6181.

Arts M.J.T.J., Haenen G.R.M.M., Voss H.-P., Bast A., Antioxidant capacity of reaction products limits the applicability of the Trolox Equivalent Antioxidant Capacity (TEAC) asaay. Food Chem. Toxicol. 2004; 42:45-49.

Bortolomeazzi R., Verardo G., Liessi A., Callea A., Formation of dehydrodiisoeugenol and dehydrodieugenol from the reaction of isoeugenol and eugenol with DPPH radical and their role in the radical scavenging activity. Food Chem. 2010; 118:256-265.

Bouayed J., Bohn T., Exogenous antioxidants - Double-edged swords in cellular redox state. Health beneficial effects at physiologic doses versus deleterious effects at high doses. Oxid. Med. Cell Longev. 2010; 3:228-237.

Brand-Williams W., Cuvelier M.E., Berset C., Use of a free radical method to evaluate antioxidant activity. Lebensm. Wiss. Technol. 1995; 28:25-30.

Dawidowicz A.L., Olszowy M., Mechanism change in estimating of antioxidant activity of phenolic compounds. Talanta 2012 ; 97:312-317.

Foti M.C., Daquino C., Geraci C., Electron-transfer reaction of cinnamic acids and their methyl esters with the $\mathrm{DPPH}^{\bullet}$ radical in alcoholic solutions. J. Org. Chem. 2004; 69:2309-2314.

Foti M.C., Daquino C., Mackie I.D., DiLabio G.A., Ingold K.U., Reaction of phenols with the 2,2-diphenyl-1-picrylhydrazyl radical. Kinetics and DFT calculations applied to determine $\mathrm{ArOH}-\mathrm{H}$ bond dissociation enthalpies and reaction mechanism. J. Org. Chem. 2008; 73:9270-9282.

Freidovich I., Fundamental aspects of reactive oxygen species, or what's the matter with oxygen? Ann. N. Y. Acad. Sci. 1999; 893:13-18.

Fukumoto L.R., Mazza G., Assessing antioxidant and prooxidant activities of phenolic compounds. J. Agric. Food Chem. 2000; 48:3597-3604.

Gülçin I., Antioxidant activity of food constituents: an overview. Arch. Toxicol. 2012; 86:345-391.

Jiménez A., Selga A., Torres J.L., Julià L., Reducing activity of polyphenols with stable radicals of the TTM series. Electron transfer versus H-abstraction reactions in flavan-3-ols. Org. Lett. 2004; 6:45834586 . 
Karadag A., Ozcelik B., Saner S., Review of methods to determine antioxidant capacities. Food Anal. Met. 2009; 2:41-60.

Khanan U.K.S., Oba S., Yanase E., Murakami J., Phenolic acids, flavonoids and total antioxidant capacity of selected leafy vegetables. J. Funct. Foods 2012; 4:979-989.

Kikuzaki H., Hisamoto M., Hirose K., Akiyama K., Taniguchi H., Antioxidant properties of ferulic acid and its related compounds. J. Agric. Food Chem. 2002; 50:2161-2168.

Kiliç I., Yeşilouğlou Y., Spectroscopic studies on the antioxidant activity of $p$-coumaric acid. Spectrochim. Acta Part B At. Spectrosc. 2013; 115:719-724.

Kristinová V., Mozuraityte R., Storrø I., Rustad T., Antioxidant activity of phenolic acids in lipid oxidation catalized by different prooxidants. J. Agric. Food Chem. 2009; 57:10377-10385.

Medina I., Gallardo J.M., González M.J., Lois S., Hedges N., Effect of molecular structure of phenolic families as hydroxycinnamic acids and catechins on their antioxidant effectiveness in minced fish muscle. J. Agric. Food Chem. 2007; 55:3889-3895

Nenadis N., Lazaridou O., Tsimidou M.Z., Use of reference compounds in antioxidant activity assessment. J. Agric. Food Chem. 2007; 55:5452-5460.

Nenadis N., Tsimidou M., Observations on the estimation of scavenging activity of phenolic compounds using rapid 1,1-diphenyl-2picryl-hydrazyl ( $\left.\mathrm{DPPH}^{\bullet}\right)$ tests. J. Am. Oil Chem. Soc. 2002; 79:11911195.

Nenadis N., Wang L.-F., Tsimidou M., Zhang H.-Y., Estimation of scavenging activity of phenolic compounds using the $\mathrm{ABTS}^{\bullet+}$ assay. J. Agric. Food Chem. 2004; 52:4269-4274

Neves M.A., Miguel M.G., Pedroso L.G., Barroso J.G., Figueiredo A.C., Martins D.S., Dandlen S.A., Variability of olive oil cultivar on stability during storage. Acta Aliment. 2011; 40: 436-448.

Osborn H.T., Akoh C.C., Effects of natural antioxidants on iron-catalized lipid oxidation of structured lipid-based emulsions. J. Am. Oil Chem. Soc. 2003; 80:847-852.

Pereira D.M., Valentão P., Pereira J.A., Andrade P.B., Phenolics: from chemistry to biology. Molecules 2009; 14:2202-2211.

Pérez-Jiménez J., Saura-Calixto F., Effect of solvent and certain food constituents on different antioxidant capacity assays. Food Res. Int. 2006; 39:791-800.

Prior R.L., Wu X., Schaich K., Standardized methods for the determination of antioxidant capacity and phenolics in foods and dietary supplements. J. Agric. Food Chem. 2005; 53:4290-4302.

Re R., Pellegrini N., Proteggente A., Pannala A., Yang M., Rice-Evans C. Antioxidant activity applying an improved ABTS radical cation decolorization assay. Free Radical Biol. Med. 1999; 26:1231-1237.
Rice-Evans C.A., Miller N.J., Paganga G., Structure-antioxidant activity relationships of flavonoids and phenolic acids. Free Rad. Biol. Med. 1996; 20:933-956.

Roginsky V., Lissi E.A., Review of methods to determine chain-breaking antioxidant activity in food. Food Chem. 2005; 92,235254.

Scherer R., Godoy H.T., Antioxidant activity index (AAI) by the 2,2-diphenyl-1-picrylhydrazyl method. Food Chem. 2009; 112:654658.

Stratil P., Klejdus B., Kubán V., Determination of total content of phenolic compounds and their antioxidant activity in vegetables evaluation of spectrophotometric methods. J. Agric. Food Chem. 2006; 54:607-616.

Teixeira J., Gaspar A., Garrido E.M., Garrido J., Borges F., Hydroxycinnamic acid antioxidants: an electrochemical overview. BioMed. Res. Int. 2013; Article ID251754, 11 pages.

Uttara B., Singh A.V., Zamboni P., Mahajan R.T., Oxidative stress and neurodegenerative diseases: a review of upstream and downstream antioxidant therapeutic options. Cur. Neuropharmacol. 2009; 7:65-74.

von Gadow A., Joubert E., Hansmann C.F., Comparison of the antioxidant activity of aspalathin with that of other plant phenols of rooibos tea (Aspalathus linearis), $\alpha$-tocopherol, BHT, and BHA. J. Agric. Food Chem. 1997; 45:632-638.

Xie J., Schaich K.M., Re-evaluation of the 2,2-diphenyl-1picrylhydrazyl free radical (DPPH) assay for antioxidant activity. J. Agric. Food Chem. 2014; 62:4251-4260.

Yordi E.G., Pérez E.M., Matos M.J., Villares E.U. 2012. Antioxidant and Pro-Oxidant Effects of Polyphenolic Compounds and Structure-Activity Relationship Evidence, Nutrition, Well-Being and Health, Dr. Jaouad Bouayed (Ed.). InTech.

Yoshikawa T., Free radicals and their scavengers in Parkinson's disease. Eur. Neurol. 1993; 33:60-68.

\section{How to cite this article:}

Jaqueline Badanai, Celi Silva, Denise Martins, Dulce Antunes, Maria Graça Miguel. Ability of scavenging free radicals and preventing lipid peroxidation of some phenols and ascorbic acid. J App Pharm Sci, 2015; 5 (08): 034-041. 